\title{
CYP2D6 and pharmacogenomics: where does future research need to focus? Part 2: clinical aspects
}

\author{
"...the primary challenge for future research is to step back and \\ critically assess the knowledge deficits, and design appropriate studies \\ to generate convincing evidence to justify a change in practice..."
}

Keywords: clinical implementation • CPIC • CYP2D6 • cytochrome P450

- drug-metabolizing enzymes $\bullet$ genetic variation $\bullet$ pharmacogenetics

In a previous commentary [1] we addressed the complexity of the highly polymorphic CYP2D6 locus. In this second part of the mini-series we address some issues relevant to implementation of CYP2D6 pharmacogenetics (PGx) into clinical practice and opportunities for future research.

The potential clinical impact of CYP2D 6 variation is illustrated by the inclusion of specific language regarding CYP2D6 PGx into the product labels for 36 of the 137 drugs or drug combinations listed in the US FDA Table of Pharmacogenomics Biomarkers in Drug Labeling [2]. With few exceptions (e.g., aripiprazole [3]) product labels containing language related to CYP2D6 PGx rarely contain specific recommendations with respect to how much a drug dose should be increased or decreased for a given genotype or predicted phenotype [4], despite a number of studies describing the magnitude of difference in drug exposure or drug clearance between poor metabolizer and extensive metabolizer phenotypes $[5,6]$, and efforts to consolidate data for guidance purposes $[7,8]$. Therefore, it is not surprising that sufficient evidence to recommend routine clinical implementation of a priori genotyping in support of CYP PGx testing in the psychiatric setting is lacking [9-11]. Rather than bemoan the reluctance of payers, regulators and some clinicians to embrace CYP2D6 PGx with open (and uncritical) arms, the primary challenge for future research is to step back and critically assess the knowledge deficits, and design appropriate studies to generate convincing evidence to justify a change in practice; we offer for consideration our thoughts in this regard.

The difficulty in applying the results of genome-wide association studies to individual patients is recognized - even if a genetic variant is significant by genomewide association study standards, it is difficult to know if an individual carrying the variant of interest will display the phenotype of interest. Likewise, the dose of a medication that, on average, produces a statistically significant decrease in a disease phenotype in a treated population and becomes the approved dose in the product label may not necessarily result in clinical improvement for a specific patient. PGx research seeks to identify the subset of patients that is most likely to respond to a given medication. PGx studies focusing on genes involved in drug disposition primarily involve CYPs [12] as drug clearance is a key determinant of the dose exposure component of the dose-exposure-response continuum. It is important to bear in mind that the relevant proximal phenotype for CYP allelic variants is the formation of CYP isoform-specific metabolites, and not response to the drug. Disappearance of the substrate as reflected by in vivo drug clearance is also a common

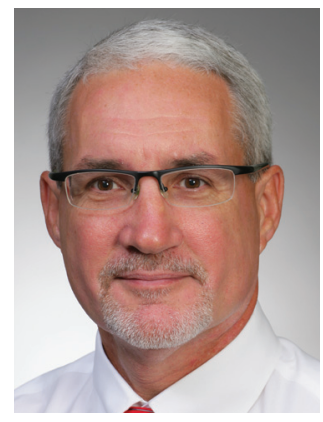

J Steven Leeder* *Author for correspondence: Division of Clinical Pharmacology, Toxicology \& Therapeutic Innovation, Department of Pediatrics, Children's Mercy Kansas City \& University of MissouriKansas City, 2401 Gillham Rd, Kansas City, MO 64108, USA Tel.: +1 8162343088 Fax: +18168551958 sleeder@cmh.edu

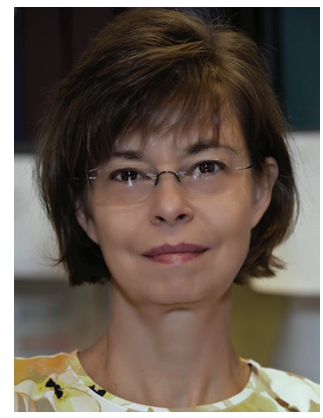

Andrea Gaedigk Division of Clinical Pharmacology, Toxicology \& Therapeutic Innovation, Department of Pediatrics, Children's Mercy Kansas City \& University of MissouriKansas City, 2401 Gillham Rd, Kansas City, MO 64108, USA

Future Medicine part of 
phenotype for allelic variation in CYP2D6 and other CYP genes. However, we often fail to properly consider some of the nuances associated with 'clearance' and 'exposure' when attempting to relate genetic variation in CYPs and other genes involved in drug disposition with drug response. The relevant drug exposure is the concentration at the site of action, which is the brain for CYP2D6 substrates such as atypical antipsychotics, selective serotonin reuptake inhibitors and tricyclic antidepressants, and breast tumor for tamoxifen. By contrast, the pharmacokinetic consequences of $C Y P 2 D 6$ genetic variation are measured in the plasma. Hence, a considerable part of the disconnect between CYP2D6 genotype and drug response lies in the myriad factors between drug concentrations in the sampled compartment - blood/plasma - and the concentration at the site of action, not to mention the variability of the drug target itself and downstream effector processes.

Administration of a fixed drug dose to a population varying in size and body composition will result in a range of drug exposures. For drugs highly dependent on CYP2D6 for their elimination, genotype represents an additional source of interindividual variability, and the magnitude of the effect is a function of the fractional contribution of CYP2D6 to total clearance; an example is atomoxetine, with a tenfold difference in area under the curve between poor and extensive metabolizers [13]. Viewed from a different perspective, if variability in drug exposure contributes to variability in drug response, is it reasonable to expect that genotype-controlled drug exposure will minimize one source of variability in drug response? Controlling drug exposure could theoretically facilitate the detection of genetic variation in genes more directly involved in drug response. Administration of the same/similar dose of a CYP2D6 substrate to a population results in a range of drug concentrations/exposures for which CYP2D 6 genotype is a primary source of the observed variability. By analogy, achieving the same or similar drug exposure in a study population has the potential to result in the identification of the major sources of variability in drug response. CYP2D6 genotype would then become one of several genetic factors contributing to the PGx profile predicting the probability of clinical response. At a minimum, future clinical studies could be designed to determine if genotype-directed dosing to a target exposure reduces the amount of time required to achieve the desired response for those drugs where the usual practice is to titrate to clinical response. This concept has been explored for tamoxifen, a drug dependent upon CYP2D6 for bioactivation to its active form, endoxifen [14].
The practice of titrating dose (adjusting drug exposure) to achieve the desired clinical response implies that a relationship exists between drug exposure and drug response. Although therapeutic drug monitoring is not routinely employed for many CYP2D6 substrates, therapeutic reference ranges have been compiled by a German working group (AGNP) [15] for many drugs used in psychiatry. Furthermore, these and more recently published data have been utilized for genotype-stratified dosing recommendations for several classes of drugs, including CYP2D6 substrates, that are used to treat neuropsychiatric disorders [8]. Future research could be directed toward validating the proposed dose adjustments for the major genotype classes to achieve the 'population' target reference range, or establish new genotypestratified dosing guidelines. Furthermore, pharmacometric modeling has been explored as a means to develop exposure-matching dosing guidelines for pimozide [16], and holds promise for further refinement of dose individualization through the inclusion of additional patient-specific variables in addition to CYP2D6 genotype.

\section{"We propose that the focus of future CYP2D6 research should shift from a debate about whether pre-emptive genotyping for a given gene-drug pair is cost effective and warranting reimbursement by third-party payers to an emphasis on providing more concrete guidance on genotype-guided dosing adjustments."}

As the AGNP expert panel cautions, however, the proposed therapeutic reference ranges represent population-based ranges and may not be applicable to all patients. On the other hand, patients who fail to respond despite having achieved a specified target drug exposure in blood/plasma may represent a subpopulation with altered transporter function affecting drug concentration at the site of action (brain, breast and tumor), or a disease subtype that is unmasked by the drug, with drug response serving as a molecular probe for disease stratification. Although it is unlikely to be the sole solution to individualizing drug therapy for CNS-active medications, it seems reasonable to expect that exploiting CYP2D6 genotype to achieve a target plasma drug exposure should result in less variability in drug concentration at the site of action than administering a fixed dose in the absence of genotype-based adjustments.

CYP2D6 is one of the more highly expressed CYPs in the brain and is expressed in an anatomically sequestered manner that implies a role in the regulation of endogenous compounds that modulate brain function [17]. 
Neuroimaging studies are also consistent with a functional role for CYP2D6 in the brain. For example, resting blood perfusion has been reported to be associated with CYP2D6 genotype [18], and it has been suggested that sustained performance in attentional tasks and vigilance may be a functional consequence of variability in brain CYP2D6 expression [19]. Thus, an intriguing area of future CYP2D6 research is the potential to exploit the potential, but as yet uncharacterized, role of CYP2D6 in mediating endogenous functions that may be relevant for disease pathogenesis and drug response; pharmacometabolomic investigations analogous to recent reports involving CYP2C19/citalopram and escitalopram [20], and CYP3A4/sertraline [21] drug pairs offer one strategy to pursue.

Optimizing the use of most medications in clinical practice is hampered by the absence of reliable biomarkers to predict which patient will respond to what medication at what dose, and CYP2D6 substrates used to treat psychiatric disorders, pain, cardiovascular disease and cancer are no exceptions. The lack of concordance among studies investigating CYP2D6 genotype as a determinant of response to tamoxifen is a particularly good example of the difficulty in translating genetic variation affecting drug biotransformation phenotype to clinical response as several additional factors may obscure the CYP2D6 genotype-tamoxifen response relationship, including tumor heterogeneity in the breast cancer population, a complex tamoxifen metabolism/bioactivation pathway that includes several phase 1 and phase 2 pathways, genotyping procedure(s), concurrent treatment with CYP2D6 inhibitors and adherence to the prescribed treatment regimen, among others [22]. The inherent

\section{References}

1 Gaedigk A, Leeder JS. CYP2D6 and pharmacogenomics: where does future research need to focus? Part 1: technical aspects. Pharmacogenomics 15(4), 407-410 (2014).

2 US FDA. Table of Pharmacogenomics Biomarkers in Drug Labeling. www.fda.gov/Drugs/ScienceResearch/ResearchAreas/ Pharmacogenetics/ucm083378.htm

3 Pharmacogenomics Knowledge Base: PharmGKB. FDA label for aripiprazole and CYP2D6. www.pharmgkb.org/label/PA166104839

4 Pharmacogenomics Knowledge Base: PharmGKB. CPIC pairs (see PGx on FDA label). www.pharmgkb.org/cpic/pairs

5 Zhou S-F. Polymorphism of human cytochrome P450 2D6 and its clinical significance: part I. Clin. Pharmacokin. 48, 689-723 (2009). challenges of such retrospective PGx biomarker studies have been clearly articulated by Province et al. [22], and yet waiting for definitive, incontrovertible 'proof' of value seems to be an inordinately high bar to achieve.

We propose that the focus of future CYP2D6 research should shift from a debate about whether preemptive genotyping for a given gene-drug pair is cost effective and warranting reimbursement by third-party payers to an emphasis on providing more concrete guidance on genotype-guided dosing adjustments. The rationale for this position is that the CYP2DG genotype needs to be determined once (given that the test was reliable and of high quality) and the information likely will be applicable to other medications the patient may receive in the future. Furthermore, an increasing number of individuals will have knowledge of their CYP2D6 and other genotypes from direct-toconsumer genetic companies, and the cost of acquiring genotype data, per se, will become less important; what will become important will be access to high-quality data with which to provide the best possible treatment options to patients and their providers. Generating these data is the challenge for the future.

\section{Financial \& competing interests disclosure}

The authors are members of the Clinical Pharmacogenetics Implementation Consortium and contribute to the development of guidelines published by this group. The authors have no other relevant affiliations or financial involvement with any organization or entity with a financial interest in or financial conflict with the subject matter or materials discussed in the manuscript apart from those disclosed.

No writing assistance was utilized in the production of this manuscript.
6

\section{Znd its clinicl significan 761-804 (2009).}

7 Kirchheiner J, Nickchen K, Bauer M et al. Pharmacogenetics of antidepresants and antipsychotics: the contribution of allelic variants to the phenotype of drug response. Mol. Psychiatr. 9, 442-473 (2004).

8 Stingl JC, Brockmöller J, Viviani R. Genetic variability of drug-metabolizing enzymes: the dual impact on psychiatric therapy and regulation of brain function. Mol. Psychiatr. 18, 273-287 (2013).

9 Evaluation of Genomic Applications in Practice and Prevention (EGAPP) Working Group. Recommendations from the EGAPP Working Group: testing for cytochrome P450 polymorphisms in adults with nonpsychotic depression treated with selective serotonin reuptake inhibitors. Genet. Med. 9, 819-825 (2007).

10 Thakur M, Grossman I, McCrory DC et al. Review of evidence for genetic testing for CYP450 polymorphisms 
in management of patients with nonpsychotic depression with selective serotonin reuptake inhibitors. Genet. Med. 9 , 826-835 (2007).

11 Grossman I, Sullivan PF, Walley N et al. Genetic determinants of variable metabolism have little impact on the clinical use of leading antipsychotics in the CATIE study. Genet. Med. 10, 720-729 (2008).

12 Holmes MV, Shah T, Vickery C, Smeeth L, Hingorani AD, Casas JP. Fulfilling the promise of personalized medicine? Systematic review and field synopsis of pharmacogenetic studies. PLoS ONE 4(12), e7960 (2009).

13 Sauer J-M, Ring BJ, Witcher JW. Clinical pharmacokinetics of atomoxetine. Clin. Pharmacokinet. 44, 571-590 (2005).

14 Irvin Jr WJ, Walko CM, Weck KE et al. Genotype-guided tamoxifen dosing increases active metabolite exposure in women with reduced CYP2D6 metabolism: a multicenter study. J. Clin. Oncol. 29, 3232-3239 (2011).

15 Hiemke C, Baumann P, Bergemann N et al. AGNP consensus guidelines for therapeutic drug monitoring in psychiatry: update 2011. Pharmacopsychiatry 44, 195-235 (2011).

16 Rogers HL, Bhattaram A, Zineh I et al. CYP2D6 genotype information to guide pimozide treatment in adult and pediatric patients: basis for the US Food and Drug
Administration's new dosing recommendations. J. Clin. Psychiatry 73, 1187-1190 (2012).

17 Cheng J, Zhen Y, Miksys S et al. Potential role of CYP2D6 in the central nervous system. Xenobiotica 43, 973-984 (2013).

18 Kirchheiner J, Seeringer A, Godoy AL et al. CYP2D6 in the brain: genotype effects on resting brain perfusion. Mol. Psychiatr. 16, 333-341 (2011).

19 Stingl JC, Esslinger $\mathrm{C}$, Tost $\mathrm{H}$ et al. Genetic variation in CYP2D6 impacts neural activation during cognitive tasks in humans. Neuroimage 59, 2818-2823 (2011).

20 Ji Y, Hebbring S, Zhu H et al. Glycine and a glycine dehydrogenase (GLDC) SNP as citalopram/escitalopram response biomarkers in depression: pharmacometabolomicsinformed pharmacogenomics. Clin. Pharmacol. Ther. 89, 97-104 (2011).

21 Kaddurah-Daouk R, Boyle SH, Matson W et al. Pretreatment metabotype as a predictor of response to sertraline or placebo in depressed outpatients: a proof of concept. Transl. Psychiatry 1, e26 (2011).

22 Province MA, Goetz MP, Brauch $\mathrm{H}$ et al. CYP2D6 genotype and adjuvant tamoxifen: meta-analysis of heterogeneous study populations. Clin. Pharmacol. Ther. 95, 216-277 (2014). 